\title{
Spectral Transforms Calculation through Decision Diagrams
}

\author{
RADOMIR S. STANKOVIĆa and BOGDAN J. FALKOWSKI ${ }^{\mathrm{b}, *}$ \\ ${ }^{a}$ Braće Taskovića 17/29, Niš 18000, Minor Yugoslavia; ${ }^{\text {} N a n y a n g ~ T e c h n o l o g i c a l ~ U n i v e r s i t y, ~ S c h o o l ~ o f ~ E l e c t r i c a l ~ a n d ~ E l e c t r o n i c ~ E n g i n e e r i n g, ~ B l o c k ~ S 1, ~}$ \\ Nanyang Avenue, Singapore, Singapore 639798
}

(Received 20 January 2000; In final form 4 October 2000)

\begin{abstract}
In this paper, calculation of spectral transforms through Decision diagrams (DDs) and relationship of this method with FFT-like algorithms is discussed. It is shown that in DDs methods the basic operations in FFT-like algorithms are performed not on vectors but instead on parts of DDs as a data structure. Such a data structure represents the input signals, the intermediate results obtained during the calculation as well as the final output results. It should be noticed that, unlike FFT-like algorithms, DDs methods permit to take advantages from both, the properties of the transform matrices and the particular properties of the processed signals.
\end{abstract}

Keywords: Spectral transforms; Kronecker transforms; Complex Hadamard transforms; Fast Fourier transform; Decision diagrams

\section{DISCRETE FUNCTIONS}

Let $G$ be a finite Abelian group of order $g$ decomposable in the form

$$
G=\times_{i=1}^{n} G_{i}, \quad g=\prod_{i=1}^{n} g_{i}
$$

Denote by $P$ a field that may be a finite (Galois) field $G F(p)$, the fields $R$ or $C$ of real and complex numbers.

A discrete function is defined as a mapping $f: G \rightarrow P$. For a given $G$ and $P$, the set $P(G)$ of such functions endorse the structure of a vector (Hilbert) space. From Eq. (1), a function $f \in P(G)$ can be considered as an $n$ variable function $f\left(x_{1}, \ldots, x_{n}\right), x_{i} \in G_{i}$.

Switching functions are a particular example for $G=$ $C_{2}^{n}, C_{2}=(\{0,1\}, \oplus)$ and $P=G F(2)$. In this case $\oplus$ is the addition modulo 2, denoted as EXOR.

\section{DECISION DIAGRAMS}

Decision diagrams (DDs) are a data structure convenient for representation of discrete functions [5,19,28,34]. DDs are derived by the reduction of the corresponding Decision trees (DTs). The reduction is performed by sharing the isomorphic subtrees and deleting the redundant information in the DT using the suitably defined reduction rules [28].

Various DDs are defined for representation of different classes of discrete functions $[19,28]$. Probably the most widely known are the Binary decision diagrams (BDDs) $[1,4]$, for representation of switching functions. For a given $f$, the BDD is derived by the reduction of the corresponding Binary decision tree (BDT) [28].

BDT for switching functions of a given number of variables can be derived by the recursive application of the Shannon decomposition rule $f=\bar{x}_{i} f_{0} \oplus x_{i} f_{1}$, where $f_{0}=$ $f\left(x_{i}=0\right)$ and $f_{1}=f\left(x_{i}=1\right)$ to all the variables in $f$.

Example 1 Figure 1 shows BDT for $f\left(x_{1}, x_{2}, x_{3}\right)$ given by the truth-vector $\mathbf{F}=[0,1,0,0,1,1,0,1]^{T}$. This function will be used in further examples illustrating the considerations in this paper.

In a BDT for $f$, some isomorphic subtrees may appear. They correspond to some equal subvectors or the constant subvectors of orders $2^{k}, k \leq n-1$, in $\mathbf{F}$. Thanks to that, BDT can be reduced into a BDD for $f$. In the first case, the subtrees may be shared and the redundant information deleted from the BDT. In the second case, the subtree reduces to a single node whose all outgoing edges point to the same constant node. Thus, it can be deleted and its input edge connected directly to the corresponding constant node. The impact of the deleted nodes can be

*Corresponding author. 


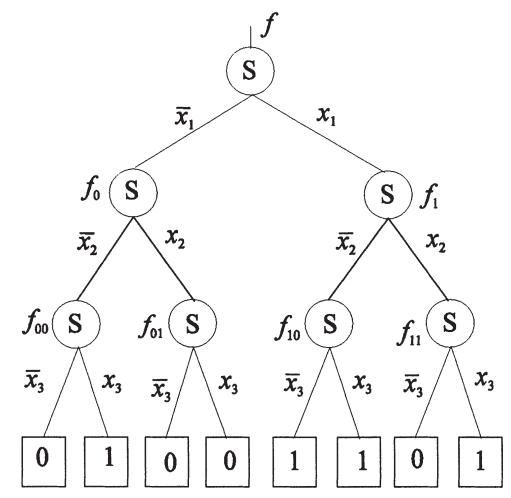

FIGURE 1 Shannon decision tree.

represented by the cross points defined as points where an edge crosses a level in the BDD. In a BDD, a level consists of nodes corresponding to the same variable. As example, two cross points are shown in Fig. 2.

Example 2 In $\mathbf{F}$ for $f$ in Example 1, there are two equal subvectors of order 2 and two constant vectors of order 2. Figure 2 shows BDD for $f$ derived by using these properties off.

In the Shannon decomposition rule, no restrictions are posed on the values that $f$ can take. Thus, $\oplus$ may be replaced by the ordinary addition in $C$, and the BDDs may be generalized into the Multi-Terminal Binary DDs (MTBDDs) used to represent the complex-valued functions on $C_{2}^{n}$ [7,8]. Extension of the concept to DDs for functions on groups of the form (1) is straightforward. It is done by using the non-terminal nodes at the $i$-th level with $g_{i}$ outgoing edges. Such DDs are denoted as Multiterminal DDs (MTDDs).

\section{SPECTRAL TRANSFORMS}

A set $Q$ of $g$ linearly independent functions on $G$ into $P$ form a basis in $P(G)$. Thus, any $f \in P(G)$ can be represented as a linear combination of basic functions from $Q$. We denote by $\mathbf{Q}(n)$ a $(g \times g)$ matrix whose columns are elements of $\mathbf{Q}$.

Definition 1 For $f \in P(G)$ represented by a vector $\mathbf{F}=[f(0), \ldots, f(g-1)]^{T}$, the $Q$-spectrum, represented by a vector, $\mathbf{Q}_{f}=\left[Q_{f}(0), \ldots, Q_{f}(g-1)\right]^{T}$ is defined by

$$
\mathbf{Q}_{f}=\mathbf{Q}(n) \mathbf{F} .
$$

\section{Kronecker Transforms}

In practical applications some special attention has been paid to spectral transforms defined by the Kronecker product representable transforms matrices $\mathbf{Q}$, since for such transforms efficient calculation algorithms can be

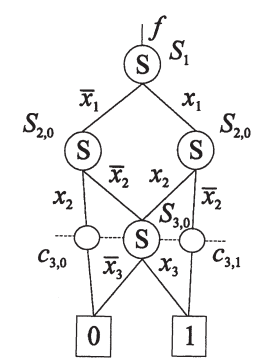

FIGURE 2 Binary decision diagram.

easily derived. These transforms may be denoted as the Kronecker transforms.

DEFINITION 2 Kronecker transforms for $f \in P(G)$ are defined by the transform matrices

$$
\mathbf{K}(n)=\bigotimes_{i=1}^{n} \mathbf{K}_{i}(1),
$$

where $\mathbf{K}_{i}(1)$ is an arbitrary $\left(g_{i} \times g_{i}\right)$ non-singular matrix with elements in $P$.

The Kronecker spectrum $K_{f}$ of a given $f \in P(G)$ is defined by $E q$. (2) if $\mathbf{Q}(n)=\mathbf{K}(n)$.

If all the submatrices $\mathbf{K}_{i}(1)=\left[k_{r, s}\right], r, s \in\left\{0, \ldots, g_{i}-\right.$ 1) are equal to each other, then $\mathbf{K}(n)$ permits the recurrence relation

$$
\mathbf{K}(n)=\left[\begin{array}{ccc}
k_{0,0} \mathbf{K}(n-1) & \ldots & k_{0, g-1} \mathbf{K}(n-1) \\
\vdots & \vdots & \vdots \\
k_{g_{i}-1,0} \mathbf{K}(n-1) & \ldots & k_{g_{i}-1, g_{i}-1} \mathbf{K}(n-1)
\end{array}\right] .
$$

Definition 2 includes some spectral transforms that are already being used in engineering practices $[21-23,33]$.

1. If $P=G F(2)$, and $\mathbf{K}_{i}=\mathbf{R}(1), \forall i$, where

$$
\mathbf{R}(1)=\left[\begin{array}{ll}
1 & 0 \\
1 & 1
\end{array}\right],
$$

the Reed-Muller transform is defined.

2. If $P=R$, and $\mathbf{K}_{i}=\mathbf{A}(1), \forall i$, where

$$
\mathbf{A}(1)=\left[\begin{array}{cc}
1 & 0 \\
-1 & 1
\end{array}\right],
$$

the arithmetic transform is defined.

3. If $P=R$, and $\mathbf{K}_{1}=\mathbf{W}(1), \forall i$, where

$$
\mathbf{W}(1)=\frac{1}{2}\left[\begin{array}{cc}
1 & 1 \\
1 & -1
\end{array}\right],
$$

the discrete Walsh transform is defined. 
4. By permitting permutation of columns [29] in $\mathbf{R}(1)$, $\mathbf{A}(1)$ and $\mathbf{W}(1)$, the multi-polarity Reed-Muller, arithmetic and Walsh transforms are defined. These transforms are discussed in detail in $[9,10,14,23]$.

\section{CHT}

Complex Hadamard Transforms (CHTs) [15,17, 18,26,27], are a broad family of spectral transforms that include several, already used, spectral transforms. At the same time, CHTs belong to the Kronecker transforms and share their useful properties, as for example, the recurrence relation (4) and fast algorithms derived from it $[16,17,26]$.

Definition 3 If $P=C$, and the constituent submatrices $\mathbf{K}_{i}(1)$ in $E q$. (3) are defined by

$$
\mathbf{K}_{i}=j^{\mathbf{A}},
$$

where $j=\sqrt{-1}$, and $\mathbf{A}$ is an $(2 \times 2)$ non-singular matrix over $G F(4)$, then the CHT are defined [15].

Example 3 If $\mathbf{A}$ is taken as the modulo 2 multiplication matrix multiplied by 2, i.e. if

$$
\mathbf{A}=\left[\begin{array}{ll}
0 & 0 \\
0 & 2
\end{array}\right],
$$

then the corresponding CHT is the Walsh transform.

Example 4 If

$$
\mathbf{A}=\left[\begin{array}{ll}
0 & 0 \\
1 & 3
\end{array}\right]
$$

the Fresnel transform associated to the Walsh transform is defined [2].

Further generalizations of CHTs are possible if different matrices $\mathbf{A}$ are chosen for any $\mathbf{K}_{i}$ in Eq. (3), or if higher dimensions for $\mathbf{A}$ are allowed.

Example 5 If $\mathbf{A}$ is taken as the modulo 4 multiplication matrix multiplied by 4 , i.e. if

$$
\mathbf{A}=\left[\begin{array}{llll}
0 & 0 & 0 & 0 \\
0 & 3 & 2 & 1 \\
0 & 2 & 0 & 2 \\
0 & 1 & 2 & 3
\end{array}\right],
$$

the complex Walsh transform matrix

$$
\mathbf{C W}(1)=\left[\begin{array}{cccc}
1 & 1 & 1 & 1 \\
1 & -i & -1 & i \\
1 & -1 & 1 & -1 \\
1 & i & -1 & -i
\end{array}\right]
$$

is defined.
In what follows, the presentation will be based on the CHT defined in the following example [16].

Example 6 If

$$
\mathbf{A}=\left[\begin{array}{ll}
0 & 1 \\
3 & 2
\end{array}\right]
$$

then

$$
\mathbf{C H T}(1)=\left[\begin{array}{cc}
1 & i \\
-i & -1
\end{array}\right] .
$$

Figure 3 shows CHT for $n=3$ determined by

$$
\mathbf{C H T}(3)=\mathbf{C H T}(1) \otimes \mathbf{C H T}(1) \otimes \mathbf{C H T}(1) .
$$

\section{FFT-LIKE ALGORITHMS}

Fast Fourier Transform (FFT) is an algorithm for efficient calculation in terms of space and time of the Discrete Fourier Transform (DFT), see for example, Refs. [3,21,22,40,41].

Extension of FFT to other Kronecker transforms is straightforward, thanks to the so-called Good-Thomas [20] factorization for transform matrices.

DEFINITION 4 Each Kronecker product representable matrix $\mathbf{K}(n)$ defined by Eq. (3) can be represented as

$$
\mathbf{K}(n)=\prod_{i=1}^{n} \mathbf{C}_{K_{i}}(n)
$$

where

$$
\mathbf{C}_{K_{i}}(n)=\bigotimes_{i=1}^{n} \mathbf{C}_{K_{i}}^{j}, \quad \mathbf{C}_{K_{i}}^{j}= \begin{cases}\mathbf{K}_{i}, & i=j, \\ \mathbf{I}_{g_{i}}, & i \neq j,\end{cases}
$$

where $\mathbf{I}_{g_{i}}$ is the identity matrix of order $g_{i}$.

Each of the matrices $\mathbf{C}_{K_{i}}$ defines the corresponding step in the FFT-like algorithm for $\mathbf{K}(n)$. This statement will be explained in the following example.

$$
\mathbf{C H T}(3)=\left[\begin{array}{rrrrrrrr}
1 & i & i & 1 & i & 1 & -1 & -i \\
-i & -1 & 1 & -i & 1 & -i & i & 1 \\
-i & 1 & -1 & -i & 1 & i & -i & 1 \\
-1 & i & i & 1 & -i & 1 & -1 & i \\
-i & 1 & 1 & i & -1 & -i & -i & 1 \\
-1 & i & -i & -1 & i & 1 & -1 & i \\
-1 & -i & i & -1 & i & -1 & 1 & i \\
i & 1 & 1 & -i & 1 & -i & -i & -1
\end{array}\right]
$$

FIGURE 3 CHT for $n=3$ and A defined by Eq. (1). 


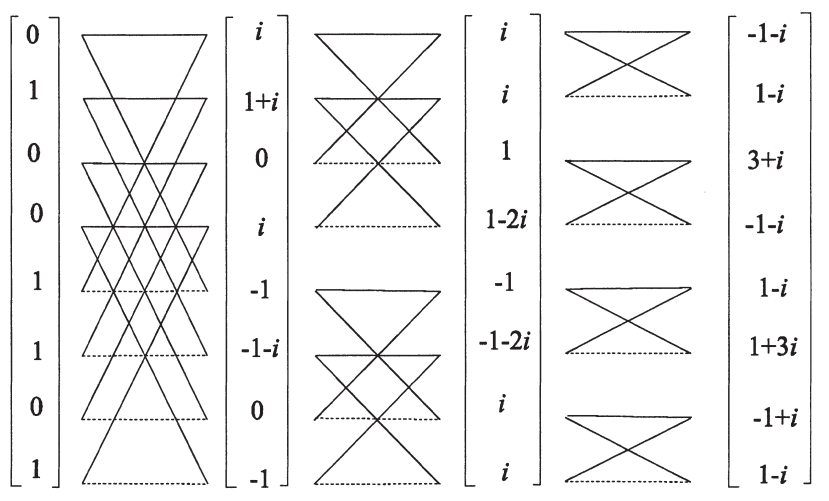

FIGURE 4 FFT-like algorithm.

Example 7 The CHT(3) matrix in Fig. 3 can be factorized as follows.

$$
\operatorname{CHT}(3)=\mathbf{C}_{1} \mathbf{C}_{2} \mathbf{C}_{3},
$$

where

$$
\begin{aligned}
& \mathbf{C}_{1}=\mathbf{C H T}(1) \otimes \mathbf{I}_{2} \otimes \mathbf{I}_{2}, \quad \mathbf{C}_{2}=\mathbf{I}_{2} \otimes \mathbf{C H T}(1) \otimes \mathbf{I}_{2}, \\
& \mathbf{C}_{3}=\mathbf{I}_{2} \otimes \mathbf{I}_{2} \otimes \mathbf{C H T}(1) .
\end{aligned}
$$

Figure 4 shows the FFT-like algorithm designed from this factorization. Each step corresponds to a matrix $\mathbf{C}_{i}$, $i=1,2,3$, and performs calculations shown by the nonzero elements in it.

Major disadvantage of FFT-like algorithms for spectral transform calculations is their exponential complexity in both space and time. For functions on an Abelian group of the form (1), the time complexity of FFT-like algorithms is $O(n g)$. The space complexity approximates to $O(g)$ provided all the calculations are done in-place. Such implementations are possible, since the input in the $i$-th step is the output from the $(i-1)$-th step of the algorithm and the values calculated in the previous steps are not required.

\section{FFT in Multi-processors Environment}

From Fig. 4, it is obvious that the calculation of the CHTspectrum consists of the repeated application of the same basic operation determined by CHT(1) performed in each step over a different subset of data. In a multiprocessor environment, it is most convenient if each processor performs this basic operation over the neighboring data. Therefore, in Fig. 4, the input data for each step should be reordered. Figure 5 shows such a realization for CHT(3).

\section{DD BASED CALCULATIONS}

Calculations in Figs. 4 and 5 differ in the ordering of the elements generated after each step of the algorithm. The

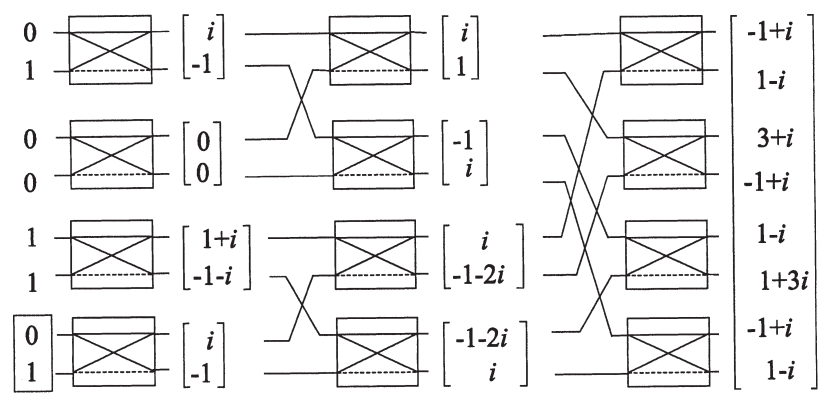

FIGURE 5 FFT-like algorithm with calculations in the multiprocessor environment.

ordering corresponds to the way in which the input data for each step are reordered.

If the operations over vectors are provided, the calculations may be further reorganized. In that case, the calculation procedure may be organized to correspond to the structure of DT representing $f$.

Example 8 Figure 6 shows the calculation of $\mathbf{C H T}(3)$ for $f$ in Example 1 through BDT in Fig. 1. Calculations are performed over the subvectors to which the outgoing edges of non-terminal nodes point to.

Similarly, as in Figs. 4 and 5, the calculations over equal subvectors are repeated in Fig. 6. Also, the constant vector whose elements are all equal to zero, is processed in the same way as any other subvector in $\mathbf{F}$ for $f$. In the BDD for $f$, such information is removed by the reduction rules. Therefore, by providing vector operations, further simplifications in the calculation procedure in Fig. 6 may be reached if it is based on the BDD instead of BDT for $f$. That is possible, since these rules do not reduce the information content of a BDT and the contribution of the deleted nodes to the spectrum of $f$ is taken into account through the cross points in the BDD.

Example 9 The CHT-spectrum for fin Example 1 can be calculated if at each node and the cross point in the BDD for $f$ in Fig. 2 the operations defined by CHT(1) are performed. In the matrix notation, the calculation procedure is described as follows.

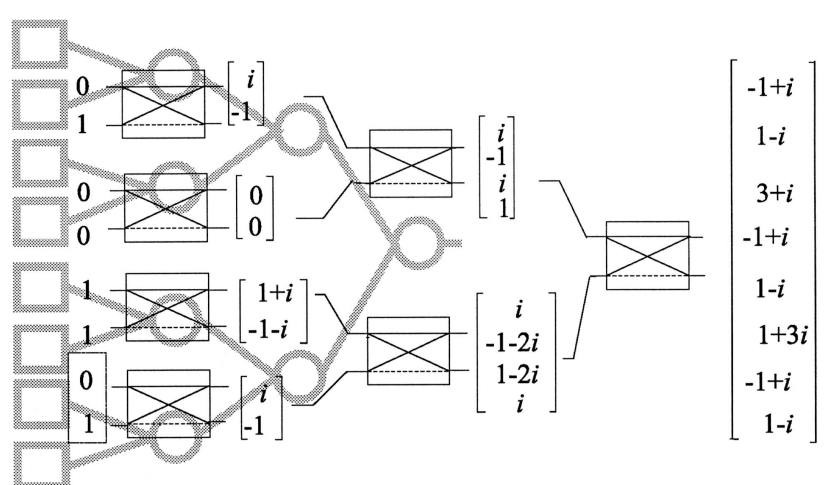

FIGURE 6 FFT-like algorithm with calculations over vectors. 
First, the node and cross points at the level corresponding to $x_{3}$ are processed, which produces:

$$
\begin{aligned}
\mathbf{C H T}_{c_{3,0}}= & {\left[\begin{array}{c}
1 \cdot 0+i \cdot 0 \\
-i \cdot 0-1 \cdot 0
\end{array}\right]=\left[\begin{array}{l}
0 \\
0
\end{array}\right], } \\
\mathbf{C H T}_{S_{3,0}} & =\left[\begin{array}{c}
1 \cdot 0+i \cdot 1 \\
-i \cdot 0-1 \cdot 1
\end{array}\right]=\left[\begin{array}{c}
i \\
-1
\end{array}\right], \\
\mathbf{C H T}_{c_{3,1}} & =\left[\begin{array}{c}
1 \cdot 1+i \cdot 1 \\
-i \cdot 1-1 \cdot 1
\end{array}\right]=\left[\begin{array}{c}
1+i \\
-1-i
\end{array}\right] .
\end{aligned}
$$

Performing CHT(1) at the nodes at the level corresponding to $x_{2}$, i.e. over the subvectors where points the outgoing edges of these nodes, we get

$$
\begin{aligned}
& \mathbf{C H T}_{S_{2,0}}=\left[\begin{array}{c}
1 \cdot\left[\begin{array}{c}
i \\
-1
\end{array}\right]+i \cdot\left[\begin{array}{l}
0 \\
0
\end{array}\right] \\
-i \cdot\left[\begin{array}{c}
i \\
-1
\end{array}\right]-1 \cdot\left[\begin{array}{l}
0 \\
0
\end{array}\right]
\end{array}\right]=\left[\begin{array}{llll}
i & -1 & 1 & i
\end{array}\right]^{T}, \\
& \mathbf{C H T}_{S_{2,1}}=\left[\begin{array}{c}
1 \cdot\left[\begin{array}{c}
1+i \\
-1-i
\end{array}\right]+i \cdot\left[\begin{array}{c}
i \\
-1
\end{array}\right] \\
-i \cdot\left[\begin{array}{c}
1+i \\
-1-i
\end{array}\right]-1 \cdot\left[\begin{array}{c}
i \\
-1
\end{array}\right]
\end{array}\right]=\left[\begin{array}{llll}
i & -1-2 i & 1-2 i & i
\end{array}\right]^{T} .
\end{aligned}
$$

Performing CHT(1) at the root node we get the CHTspectrum of $f$ as follows

$$
\mathrm{CHT}_{S_{1,0}}=\left[\begin{array}{c}
1 \cdot\left[\begin{array}{c}
i \\
-1 \\
1 \\
i
\end{array}\right]+i \cdot\left[\begin{array}{c}
i \\
-1-2 i \\
1-2 i \\
i
\end{array}\right] \\
\left.-i \cdot\left[\begin{array}{c}
i \\
-1 \\
1 \\
i
\end{array}\right]-1 \cdot\left[\begin{array}{c}
i \\
-1-2 i \\
1-2 i \\
i
\end{array}\right]\right]=\left[\begin{array}{c}
-1+i \\
1-i \\
3+i \\
-1+i \\
1-i \\
1+3 i \\
-1+i \\
1-i
\end{array}\right] .
\end{array}\right.
$$

The calculation procedure described in the above example can be represented through DD as shown in Fig. 7. It is suitable for coding in a programming language.

From the above discussed example, the following Calculation procedure for the Kronecker transforms (CPKT) can be formulated:

\section{Calculation procedure CPKT}

1. Given a function $f$ by its DD. Descend DD level by level by starting from the constant nodes.

2. Perform at each non-terminal node and the cross point at the $i$-th level the operations determined by the submatrix $\mathbf{K}_{i}(1)$ over the subvectors to which point the outgoing edges of the nodes and cross points.

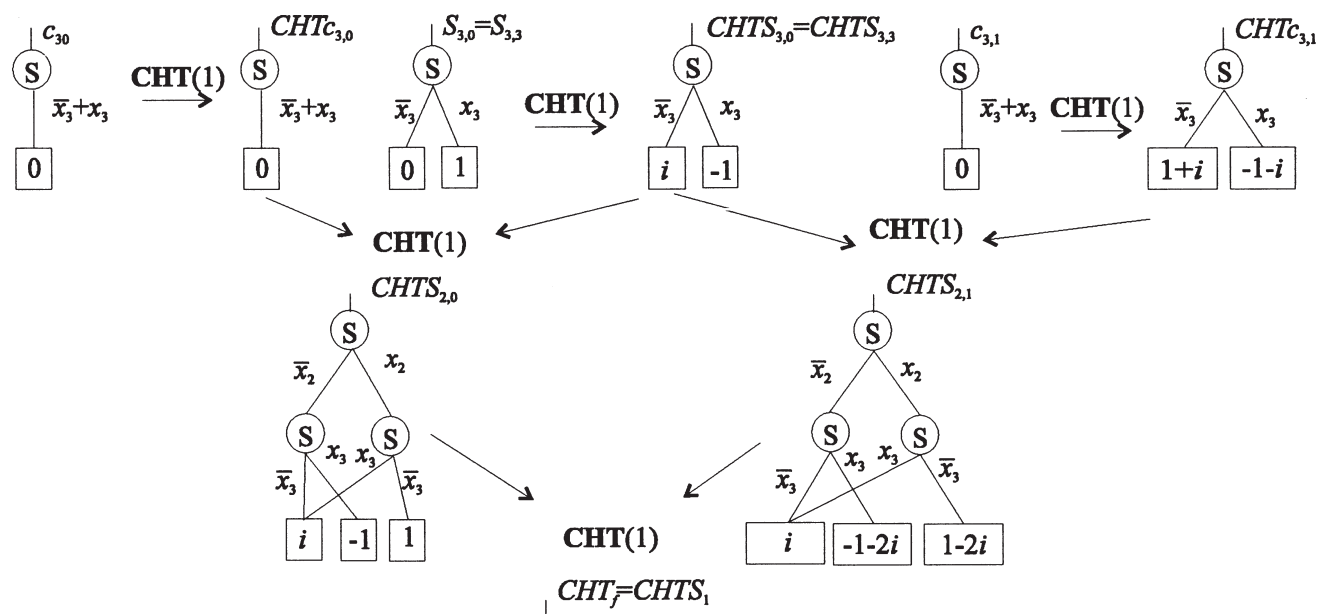

(S)

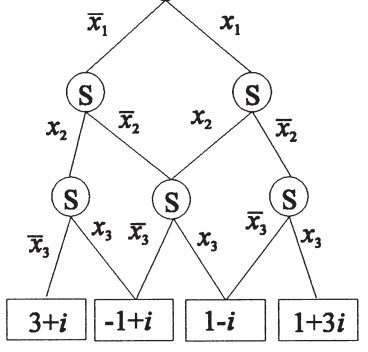

FIGURE 7 Generation of the MTBDD for CHT-spectrum. 
TABLE I Complexity of $f$ and CPU-times

\begin{tabular}{lrrrrrr}
\hline & & & & & \multicolumn{2}{c}{$t(\mathrm{msec})$} \\
$f$ & $i$ & $o$ & $s$ & $w$ & FFT & DDs \\
\hline 5xp1 & 7 & 10 & 88 & 25 & 62 & 30 \\
rd53 & 5 & 3 & 23 & 6 & 5 & 5 \\
rd84 & 8 & 4 & 59 & 11 & 157 & 30 \\
sao2 & 10 & 4 & 154 & 33 & 2309 & 170 \\
9sym & 9 & 1 & 33 & 6 & 564 & 20 \\
\hline
\end{tabular}

\section{EXPERIMENTAL RESULTS}

Similar procedure applies also to the more general multi-polarity $\mathrm{CHT}$ in which matrices $\mathbf{K}_{i}(1)$ have two types corresponding to the polarity bit 0 and 1 accordingly [5].

Table I shows complexity of BDDs for some benchmark function and CPU-times for calculation of CHT in Example 4. The number of inputs $(i)$, outputs $(o)$, size $(s)$ and width $(w)$ of DDs are shown. Calculation time is given in milliseconds. Table II shows complexity of the produced MTBDDs for CHT spectrum of these functions. In this case, the number of non-terminal nodes (ntn) and constant nodes (cn) are given, since in CHTDDs the constant nodes are complex-valued. This table shows that for some functions CHTDDs provide more compact representations than BDDs. As for any other DD, there are the opposite examples. Calculations are performed on a $133 \mathrm{MHz}$ Pentium PC with a $32 \mathrm{MB}$ RAM.

\section{FFT-LIKE ALGORITHMS VERSUS DD METHODS}

Basic characteristics of FFT-like algorithms are the following.

1. FFT-like algorithms are based upon the decomposition of the transform matrix $\mathbf{K}(n)$ into the product of $n$ sparse matrices $\mathbf{K}(n)=\mathbf{C}_{K_{1}} \ldots \mathbf{C}_{K_{n}}$. Each $\mathbf{C}_{K_{i}}$ describes a step in the algorithm. Decomposition is possible, thanks to the recursive structure of $\mathbf{K}(n)$ originating in the assumed decomposition for $G$ and the way of definition of the basis represented by columns of $\mathbf{K}^{-1}$.

2. The calculations are performed over the vector representations of the input data $\mathbf{F}$ and the intermediate results $\mathbf{F}_{i}, i=1, \ldots, n-1$, after each step, producing finally $\mathbf{S}_{f}=\mathbf{F}_{n}$.

TABLE II Complexity of CHTDDs.

\begin{tabular}{lrrrr}
\hline$f$ & ntn & cn & \multicolumn{1}{c}{$s$} & $w$ \\
\hline 5xp1 & 127 & 128 & 256 & 255 \\
rd53 & 15 & 6 & 21 & 5 \\
rd84 & 36 & 9 & 45 & 8 \\
sao2 & 965 & 906 & 1871 & 454 \\
9sym & 43 & 6 & 49 & 8 \\
\hline
\end{tabular}

3. Although permit parallelization within steps, FFT-like algorithms are essentially sequential algorithms, since the output of the $i$-th step is the input for the $(i+1)$-st step.

4. For a given $\mathbf{K}(n)$, and the fixed decomposition of it, the calculations of spectra for different signals are performed in the identical way, although $\mathbf{F}$ may have some particular properties, as for example, many zero elements, many identical or constant subvectors, etc. Thus, FFT-like algorithms do not exploit peculiar properties which $\mathbf{F}$ may have.

5. Complexity of calculations approximates to $O(g)$ in terms of space, providing that calculations in-place are provided, and $O(n g)$ in terms of time.

Identical calculations without exploiting possible properties of $f$ and the exponential calculation complexity may be considered as disadvantages of FFT-like algorithms limiting their applications when processing of signals defined in a large number of points is required.

Basic characteristics of DDs methods are the following.

1. Recursive structure of DDs for functions on group $G$ originates in the assumed decomposition of $G$. Since the same decomposition is assumed in FFT-like algorithms, the recursive structure of DTs matches the recursive structure of the decomposable transform matrices $\mathbf{K}$.

2. Reduction of a DT representing a given $f$ into the corresponding DD for $f$ is possible if $f$ has some properties mentioned in property 4 of FFT-like algorithms, which are not exploited in FFT-like algorithms. Thus, instead of vectors, DDs are used as a structure for data representation in calculation of spectral transforms, some advantages may be taken from such properties of $f$ permitting reduction of complexity of calculations.

3. In DDs, constant subvectors in $\mathbf{F}$ are represented by a single constant node. Equal subvectors are represented by the isomorphic subtrees, which are shared and the redundant subtrees are removed from the DD. Therefore, in DDs methods, the processing of constant subvectors is greatly simplified, and there is no repeated processing of identical subvectors in $\mathbf{F}$.

4. Besides properties of $f$, in DDs methods, we may also use peculiar properties of $\mathbf{K}(n)$ to improve the efficiency of computation, in the same way as it is done in non-constant geometry FFT-like algorithms. In these algorithms, $\mathbf{C}_{K_{i}} \neq \mathbf{C}_{K_{j}}$ for $i \neq j$. The properties of $\mathbf{C}_{K_{j}}$ may be used to reduce the number of calculations in the $j$-th step. For example, unlike the FFT for the Walsh transform, the number of calculations in steps of the non-constant geometry FFT for the Haar transform decreases when $i$ increases. The same property was used in [35] to improve efficiency of calculation of the Haar transform through DDs. 
From this consideration, the following remark is possible.

Remark 1 In FFT-like algorithms, efficiency of calculations is achieved by taking advantage of the properties of transform matrices decomposable into the product of sparse matrices. These matrices are further expressed in terms of some basic transform matrices, see for example, Refs. [40,41].

In DDs methods, the advantages are taken from both the properties of the transform matrices in the same way as in FFT-like algorithms, and the properties of the processed functions.

Therefore, for a given $G$ and fixed decomposition of $G$, FFT-like algorithm for any $f$ on $G$ has the same complexity in terms of both space and time. However, in DDs methods, complexity of calculation strongly depends on the complexity of the DD for $f$. Thanks to that, DDs permit efficient processing when $G$ is large and extend the application of spectral techniques to areas where, for the complexity, the use of FFT-like algorithms is impossible.

\section{GENERALIZATIONS}

An $n$-variable multiple-output switching function $f_{q}$ with $q$ outputs can be represented by an integer-valued function $f_{q}(z)$ determined by adding the outputs of $f_{q}$ multiplied by the weighting coefficients $2^{i}, i=0, \ldots, q-1$. Thanks to that, $f_{q}$ can be represented by a MTBDD and its spectrum for a spectral transform can be calculated by using the procedure CPKT.

If $f_{q}$ is represented by a shared BDD (SBDDs) [28] the calculation of a linear spectral transform may be performed over SBDD. The resulting spectrum for $f_{q}(z)$ is determined by adding the outputs at the root nodes of SBDD multiplied by $2^{i}$.

Several variants of BDDs bases calculation procedures for spectral transform and their extensions of the DDs based calculation methods to other transforms for switching functions are considered in several papers by different authors [5-7,11-13,19,34,35].

The method is also adapted to the so-called Edgevalued binary DDs (EVBDDs) [24] and their generalizations Factored edge-valued binary DDs (FEVBDDs) [39].

Generalizations to spectral transforms and differential operators for multiple-valued function and for functions on finite, not necessarily Abelian, groups into different fields are also provided [25,30,36-38].

\section{CLOSING REMARKS}

In this paper, an efficient symbolic algorithm for the calculation of spectral transforms on finite Abelian groups has been presented. Relationships between standard FFT-type of fast transforms calculations and DDs based calculations have been explained.

As an example, in FFT type calculations through DDs, the Kronecker product based transform called the Complex Hadamard transform was used. Such a transform operates on complex numbers and the advantages of its CHTDDs over standard BDDs for some standard benchmark functions have been shown.

Presented results can be extended to compute other spectral transform on finite Abelian groups. Extensions to computation of the Fourier transform on non-Abelian groups and Generalized Fourier transforms on these groups with generalizations defined through FFT, and complacent Gibbs derivatives on non-Abelian groups are given in $[31,32]$.

\section{References}

[1] Ackers, S.B. (1978) "Binary decision diagrams", IEEE Trans. Comput. C-27(6), 509-516.

[2] Aizenberg, N.N., Nutakov, V.D., Krenkelj, T.E. and Harbat, Ya.G. (1984) "Fresnel functions and Fresnel transforms for linear nonsingular transforms", Radiotehnika Elektronika 4, 698-704.

[3] Agaian, S., Astola, J. and Egiazarian, K. (1995) Binary Polynomial Transforms and Nonlinear Digital Filters (Marcel Dekker, New York).

[4] Bryant, R.E. (1986) "Graph-based algorithms for Boolean functions manipulation", IEEE Trans. Comput. C-35(8), 667-691.

[5] Chang, C.H. and Falkowski, B.J. (1997) "In-place transformation of generalized and complex spectra through algebraic decision diagrams", Proc. 1st IEEE Int. Conf. Inf., Commun. Signal Proce., Singapore 1, 256-260.

[6] Clarke, E.M., Zhao, X., Fujita, M., Matsunaga, Y. and McGeer, R. (1993) "Fast Walsh transform computation with binary decision diagram", In: Kebschull, U., Schubert, E. and Rosenstiel, W., eds, Proceedings of the IFIP WG 10.5 Workshop on Applications of the Reed-Muller Expansion in Circuit Design, 16-17.9.1993, Hamburg, Germany, pp 82-85.

[7] Clarke, E.M., Fujita, M. and Zhao, X. (1996) "Multi-terminal decision diagrams and hybrid decision diagrams", In: Sasao, T. and Fujita, M., eds, Representations of Discrete Functions (Kluwer Academic Publishers, Dordrecht), pp 93-108.

[8] Clarke, E.M., Fujita, M. and Heinle, W. (1997) "Hybrid spectral transform diagrams", Proc. 1st IEEE Int. Conf. Inf., Commun. Signal Process., Singapore 1, 251-255.

[9] Falkowski, B.J. (1996) "Generalized multi-polarity Hadamard Walsh transforms", In: Stanković, R.S., Stojić, M.R. and Stanković, M.S, eds, Recent Developments in Abstract Harmonic Analysis with Applications in Signal Processing (Nauka, Belgrade), pp 269-279.

[10] Falkowski, B.J. (1994) "Properties and ways of calculation of multipolarity generalized Walsh transform", IEEE Trans. Circuits Syst.II: Analog Digital Signal Process. 41(6), 380-391.

[11] Falkowski, B.J. and Chang, C.H. (1994) "Efficient algorithms for the calculation of Walsh spectrum from OBDD and synthesis of OBDD from Walsh spectrum for incompletely specified Boolean functions", Proceedings of the 37th Midwest Symposium on Circuits and Systems, 3-5.8.1994, Lafayette, LA, USA.

[12] Falkowski, B.J. and Chang, C.H. (1994) "Efficient algorithm for the calculation of arithmetic spectrum from OBDD and synthesis of OBDD from arithmetic spectrum for incompletely specified Boolean functions", Proc. 27th IEEE Int. Symp. Circuits Syst., London, UK 1, 197-200.

[13] Falkowski, B.J. and Chang, C.H. (1994) "Efficient algorithm for forward and inverse transformations between Haar spectrum and binary decision diagrams", Proc. 13th IEEE Int. Phoenix Conference on Computers and Communication, Phoenix, Arizona, April 1994, pp 497-503.

[14] Falkowski, B.J. and Chang, C.H. (1995) "Fast generalized arithmetic and adding transforms", Proc. Asia South Pacific Design Automatic Conference '95, Chiba, Japan, 723-728. 
[15] Falkowski, B.J. and Rahardja, S. (1996) "Composite complex Hadamard spectra of Boolean functions", Proc. 29th IEEE Int. Symp. Circuits Syst., Atlanta, GA, USA 4, 392-395.

[16] Falkowski, B.J. and Rahardja, S. (1996) "Complex spectral decision diagrams", Proc. 26th IEEE Int. Symp. Multiple-Valued Logic, May 29-31, 1996, Santiago de Campostela, Spain, pp 255-260.

[17] Falkowski, B.J. and Rahardja, S. (1997) "Properties and applications of Unified Complex Hadamard transforms", Proc. 27th IEEE Int. Symp. on Multiple-Valued Logic, Antigonish, Nova Scotia, Canada, May 1997, pp 131-136.

[18] Falkowski, B.J. and Rahardja, S. (1997) "Complex spectral transforms for classification of switching functions", Proc. Int Symp. IC Technol., Syst. Appl., (7th ISIC), Singapore September, 505-508.

[19] Falkowski, B.J. and Stanković, R.S. (1997) "Decision diagrams for representations of discrete functions in VLSI computer-aided design systems", Proc. Int. Symp. IC Technol., Syst. Appl., (7th ISIC), Singapore September, 86-93, Invited paper.

[20] Good, I.J. (1958) "The interaction algorithm and practical Fourier analysis", J. Roy. Statist. Soc., ser. B 20, 361-372, Addendum, 22 (1960) 372-375.

[21] Hurst, S.L., Miller, D.M. and Muzio, J.C. (1985) Spectral Techniques in Digital Logic (Academic Press, Bristol).

[22] Karpovsky, M.G. (1976) Finite Orthogonal Series in the Design of Digital Devices (Wiley/JUP, New York/Jerusalem).

[23] Kukharev, G.A., Shmerko, V.P. and Yanushkievich, S.N. (1991) Technique of Binary Data Parallel Processing for VLSI (Vysheyshaja shcola, Minsk, Belarus).

[24] Lai, Y.F., Pedram, M. and Vrudhula, S.B.K. (1994) "EVBDD-based algorithms for integer linear programming, spectral transformation, and functional decomposition”, IEEE Trans. Comput.-Aided Des. Integrated Circuits Syst. 13(8), 959-975.

[25] Miller, D.M. (1994) "Spectral transformation of multiple-valued decision diagrams", Proceedings of the 24th IEEE Int. Symp. on Multiple-valued Logic, Boston, MA, USA, 22-25.5.1994, pp 89-96.

[26] Rahardja, S. and Falkowski, B.J. (1997) "Family of complex Hadamard transforms: relationships with other transforms and complex composite spectra", Proc. 27th IEEE Int. Symp. on Multiple-Valued Logic, Antigonish, Nova Scotia, Canada May, $125-130$.

[27] Rahardja, S. and Falkowski, B.J. (1997) "Detection of Boolean symmetries using complex Hadamard transformation", Proc. 30th IEEE Int. Symp. Circuits Syst., Hong Kong June, 2116-2119.

[28] Sasao, T., Fujita, M., (1996) In: Representations of Discrete Functions (Kluwer Academic Publishers, Dordrecht).

[29] Stanković, R.S. (1982) "A note on the relation between ReedMuller expansions and Walsh transform", IEEE Trans. Electromagnetic Compatibility EMC-24(1), 68-70.

[30] Stanković, R.S. (1996) "Fast Fourier transform on finite nonAbelian groups", In: Stanković, R.S., Stojić, M.R. and Stanković, M.S., eds, Recent Developments in Abstract Harmonic Analysis with Applications in Signal Processing (Nauka, Belgrade), pp 405-420.

[31] Stanković, R.S. (1999) "Calculation of Fourier transform on finite non-Abelian groups through decision diagrams", Proc. Second International Workshop on Spectral Transforms and Filter Banks, Brandenburg, Germany, March 5-7, 1999.

[32] Stankovic, R.S., Moraga, C., Astola, J.T. "Readings in Fourier analysis on finite non-Abelian groups", TICSP Series \#5, September 1999.

[33] Varma, D. and Trachtenberg, E.A. (1993) "Efficient spectral techniques for logic synthesis", In: Sasao, T., ed, Logic Synthesis and Optimization (Kluwer Academic Publishers, Boston), pp 215-232.

[34] Stankovíc, R.S. and Falkowski, B.J. (1997) "FFT and decision diagrams based methods for calculation of spectral transforms", Proc. 1st IEEE Int. Conf. on Information, Communications and Signal Processing, Singapore Vol. 1, pp 241-245.

[35] Stanković, M., Janković, D. and Stanković, R.S. (1996) "Efficient algorithms for Haar spectrum calculation", Scientific review 21-22, $171-182$

[36] Stanković, R.S., Stanković, M., Moraga, C. and Sasao, T. (1994) "Calculation of Vilenkin-Chrestenson transform coefficients of multiple-valued functions through multiple-place decision diagrams", Proc. 5th Int. Workshop on Spectral Techniques, March 15-17, 1994, Beijing, China, pp 107-116.

[37] Stanković, R.S., Stanković, M., Moraga, C. and Sasao, T. (1994) "Calculation of Reed-Muller-Fourier coefficients of multiplevalued functions through multiple-place decision diagrams", Proc. 24th IEEE Int. Symp. on Multiple-Valued Logic, Boston, Massachusetts, USA, May 1994.

[38] Stanković, R.S., Stanković, M., Janković, D., Shmerko, V.P. and Yanushkevich, B. (1997) "Calculation of logic derivatives through decision diagrams", Proc. 6th Workshop on Post-Binary Ultra Large Scale Integration May 27, 1997, Nova Scotia, Canada, pp 53-54.

[39] Vrudhula, S.B.K., Pedram, M. and Lai, Y.-T. (1996) "Edge valued binary decision diagrams", In: Sasao, T. and Fujita, M., eds, Representations of Discrete Functions (Kluwer Academic Publishers, Dordrecht), pp 109-132.

[40] Yaroslavsky, L. (1985) Digital Picture Processing, an Introduction (Springer, Heidelberg)

[41] Yaroslavsky, L. "Transforms in nature and computers: origin, discrete representation, synthesis and fast algorithms". Proc. First Int. Workshop on Transforms and Filter banks, TICSP Series, No. 1, June 1998, pp. 3-29.

\section{Authors' Biographies}

Radomir S. Stanković received BE degree in Electronic Engineering from Faculty of Electronics, University of Niš, in 1976, and MSc, and $\mathrm{PhD}$ degrees in Applied Mathematics from Faculty of Electrical Engineering, University of Belgrade, in 1984, and 1986, respectively. He was with High School of Electrotechnic, Niš, from 1976 to 1987. From 1987 to date he is with Faculty of Electronics, Niš. Presently, he is a Professor teaching Logic Design. His research interests include switching theory and multiple-valued logic, signal processing and spectral techniques. He served as the co-editor and editor of two editorials and the author of couple of monographs in spectral techniques.

Bogdan J. Falkowski received the MSEE degree from the Technical University of Warsaw, Poland, and the PhD degree form Portland State University, Oregon, USA. His industrial experience includes research and development positions at several companies from 1978 to 1986 . He then joined the Electrical Engineering Department at Portland State University. Currently, he is an Associate professor with the School of Electrical and Electronic Engineering, Nanyang Technological University, Singapore, which he joined in 1992. His research interests include VLSI systems and design, synthesis and optimization of switching circuits, multiple-valued systems, design of algorithms, design automation, digital signal and image processing. He has published 3 book chapters and over 10 articles in the refereed journals and conferences. Dr Falkowski is a Senior Member of IEEE and a Member of IEEE Computer Society and IEEE Circuits and Systems Society. $\mathrm{He}$ is a member of Eta Kappa $\mathrm{Nu}$ and Pi Beta Upsilon. 

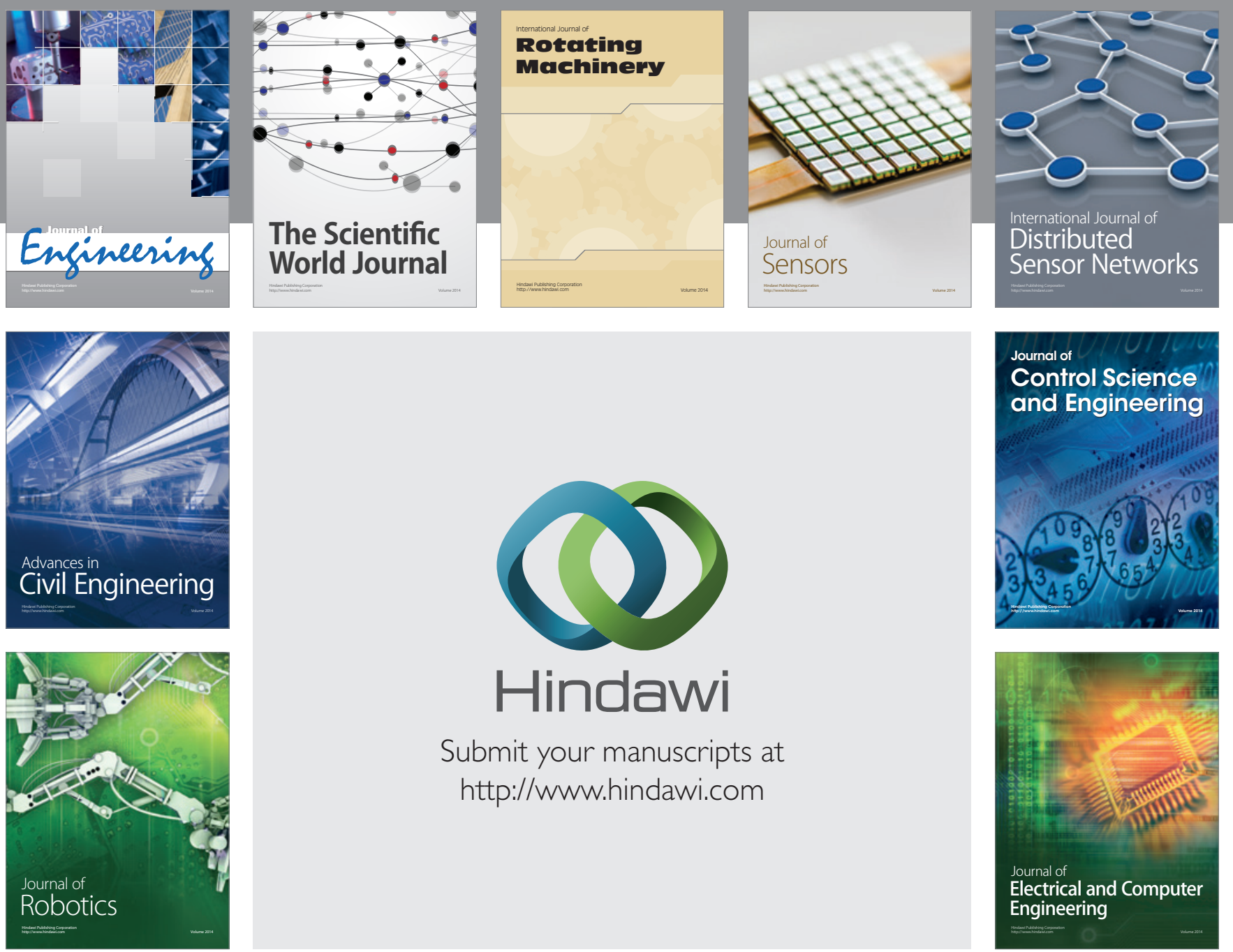

Submit your manuscripts at

http://www.hindawi.com
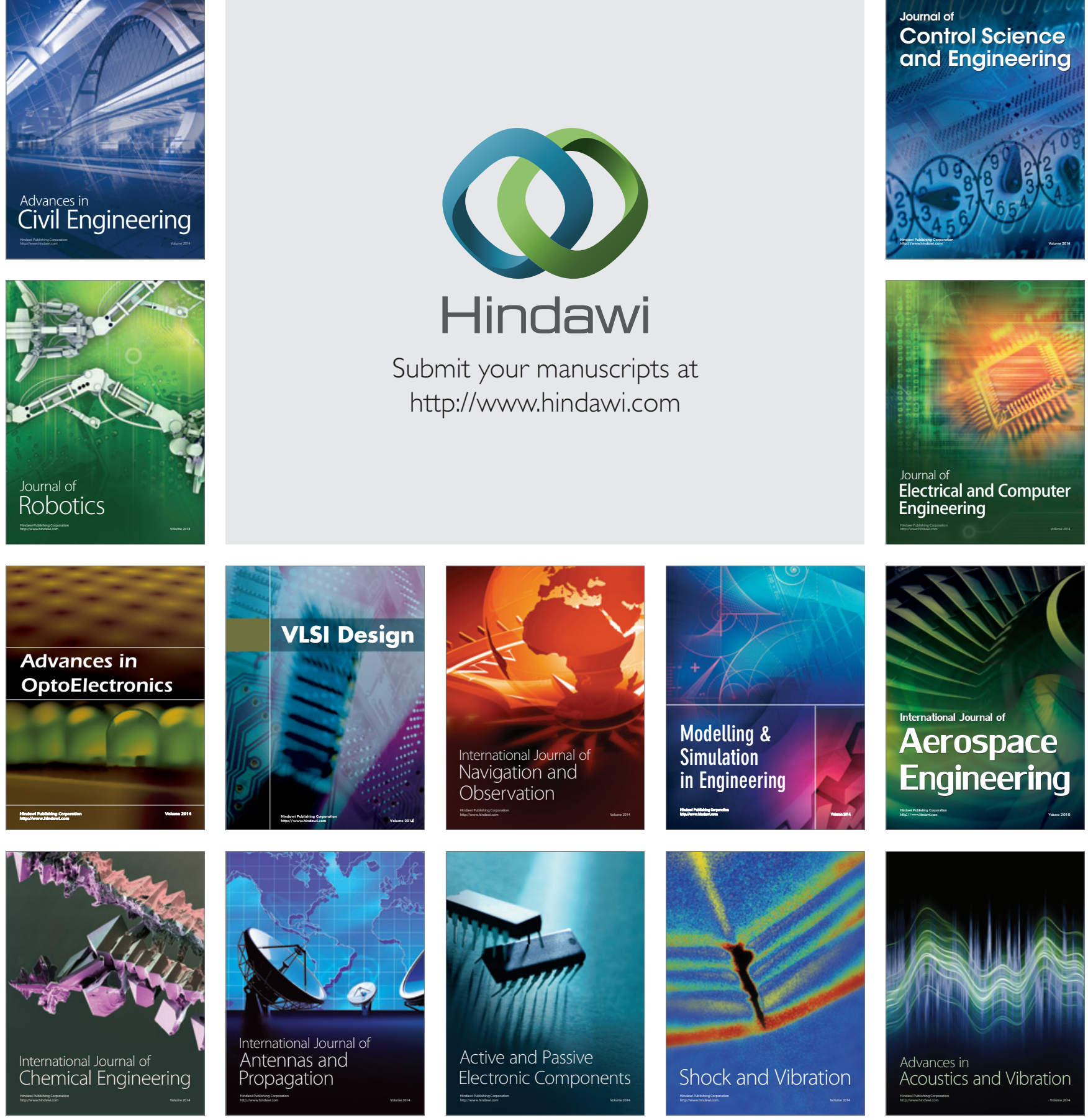